\title{
Sulfatinib, a novel kinase inhibitor, in patients with advanced solid tumors: results from a phase I study
}

\author{
Jian Ming Xu1 ${ }^{1}$, Yan Wang ${ }^{1}$, Yu Ling Chen ${ }^{1}$, Ru Jia ${ }^{1}$, Jie $\mathrm{Li}^{2}$, Ji Fang Gong ${ }^{2}$, Jing $\mathrm{Li}^{3}$, \\ Chuan $\mathrm{Qi}^{3}$, Ye $\mathrm{Hua}^{3}$, Cui Rong Tan ${ }^{3}$, Jian Wang ${ }^{4}$, Ke $\mathrm{Li}^{4}$, Yang Sai ${ }^{4}$, Feng Zhou ${ }^{5}$, \\ Yong Xin Ren ${ }^{5}$, Wei Guo Qing ${ }^{5}$, Hong Jia ${ }^{6}$, Wei Guo Su${ }^{6}$ and Lin Shen ${ }^{2}$ \\ ${ }^{1}$ Department of Gastrointestinal Oncology, The Affiliated Hospital Cancer Center (The 307th Hospital of Chinese People's \\ Liberation Army), Academy of Military Medical Sciences, Beijing, China \\ ${ }^{2}$ Department of Gastrointestinal Oncology, Peking University Cancer Hospital, Beijing, China \\ ${ }^{3}$ Clinical and Regulatory Department, Hutchison MediPharma Limited, Shanghai, China \\ ${ }^{4}$ Drug Metabolism and Pharmacokinetic Department, Hutchison MediPharma Limited, Shanghai, China \\ ${ }^{5}$ Oncology Department, Hutchison MediPharma Limited, Shanghai, China \\ ${ }^{6}$ Chemistry Department, Hutchison MediPharma Limited, Shanghai, China \\ Correspondence to: Jian Ming XU, email: jmxu2003@yahoo.com \\ Lin Shen, email: lin100@medmail.com.cn \\ Keywords: phase I clinical trial, neuroendocrine tumor, solid tumor, sulfatinib, tyrosine kinase inhibitor \\ Received: October 28, $2016 \quad$ Accepted: December 05, $2016 \quad$ Published: February 01, 2017 \\ Copyright: $\mathrm{Xu}$ et al. This is an open-access article distributed under the terms of the Creative Commons Attribution License 3.0 \\ (CC BY 3.0), which permits unrestricted use, distribution, and reproduction in any medium, provided the original author and source \\ are credited.
}

\section{ABSTRACT}

Sulfatinib is a small molecule kinase inhibitor that targets tumor angiogenesis and immune modulation. This phase I study (NCT02133157) investigated the safety, pharmacokinetic characteristics, and preliminary anti-tumor activity of sulfatinib in patients with advanced solid tumors. The study included a dose-escalation phase (50-350 $\mathrm{mg} /$ day, 28-day cycle) with a Fibonacci $(3+3)$ design, and a tumor-specific expansion phase investigating the tumor response to treatment. Two sulfatinib formulations were assessed: formulation 1 (5, 25, and $50 \mathrm{mg}$ capsules) and formulation 2 (50 and 200 mg capsules). Seventy-seven Chinese patients received oral sulfatinib; the maximum tolerated dose was not reached. Dose-limiting toxicities included abnormal hepatic function and coagulation tests, and upper gastrointestinal hemorrhage. The most common treatment-related adverse events were proteinuria, hypertension and diarrhea. Among 34 patients receiving sulfatinib formulation 2, one patient with hepatocellular carcinoma and eight with neuroendocrine tumors exhibited a partial response; 15 had stable disease. The objective response rate was $26.5 \%(9 / 34)$ and the disease control rate was $70.6 \%$ (24/34). Pharmacokinetic, safety, and efficacy data supported continuous oral administration of sulfatinib at $\mathbf{3 0 0} \mathbf{~ m g}$ as the recommended phase II dose. Sulfatinib exhibited an acceptable safety profile and encouraging antitumor activity in patients with advanced solid tumors, particularly neuroendocrine tumors.

\section{INTRODUCTION}

Vascular endothelial growth factor (VEGF)- and fibroblast growth factor (FGF)-mediated pathways play key roles in tumor angiogenesis $[1,2]$. VEGF and FGF secretion by tumor cells promotes rapid proliferation and packing of endothelial cells, which leads to the formation of excessive, coarsely packed blood vessels [3]. These blood vessels supply oxygen and nutrients to the tumor and promote tumor cell leakage into the circulation, resulting in increased tumor growth and a risk of metastasis [3]. While VEGF receptor (VEGFR)-targeted therapies are important in the management of several cancer types, many patients exhibit no or limited respond to treatment, due in part to tumor cell resistance through alternative molecular pathways [4]. 
In response to anti-VEGF therapies, some tumors can increase FGF secretion to stimulate endothelial cell proliferation, promote tumor angiogenesis, and bypass VEGF signaling pathways [4, 5]. Evidence also suggests that VEGFR, FGF receptors (FGFRs), and colony stimulating factor 1 receptor (CSF1R) promote tumor immune evasion. VEGF secreted by tumors can activate VEGFR signaling pathways in T cells; this leads to programmed cell death protein 1 (PD-1) receptor overexpression, which decreases T cell anti-tumor activity [6]. FGFR and CSF1R also appear to induce tumor-associated macrophage proliferation and differentiation, thereby promoting tumor immune evasion [7].

Targeting multiple kinases to simultaneously block VEGFR-, FGFR-, and CSF1R-mediated pathways may be a more effective method of preventing tumor angiogenesis and tumor immune evasion, and therefore represents an attractive anti-cancer therapy approach. Sulfatinib (HMPL012) is a potent small molecule tyrosine kinase inhibitor of VEGFR 1, 2, and 3, FGFR 1, and CSF1R [8], and has demonstrated selectivity in a broad kinase screening (Table 1). The aims of this phase I clinical study in patients with advanced solid tumors were to determine the sulfatinib maximum tolerated dose (MTD) and recommended dose for further phase II investigations. The study was designed to investigate the safety, pharmacokinetics (PK), and tumor response of sulfatinib.

\section{RESULTS}

\section{Patient baseline characteristics}

Seventy-seven Chinese patients were enrolled in 12 dose cohorts between April 2010 and September 2014, and followed up until July 2015 (Figure 1). The first 43 patients received sulfatinib formulation 1 and the remaining 34 received formulation 2 (Figure 2). Patient baseline demographic and clinical characteristics are summarized in Table 2.

\section{Sulfatinib exposure, dose escalation, and dose- limiting toxicities}

Sixty-six patients were enrolled in the dose-escalation phase; of these, $53(80.3 \%)$ completed the first treatment cycle. Discontinuation reasons included disease progression $(n=3)$ or deterioration $(n=1$; combined total $n=4,6.1 \%)$, consent withdrawal $(n=4,6.1 \%)$, dose-limiting toxicities (DLTs) $(n=3,4.5 \%)$, and investigator's decision $(n=2,3.0 \%)$.

In the dose-escalation phase, 43 patients received continuous treatment with sulfatinib formulation 1 at $50 \mathrm{mg}, 75 \mathrm{mg}, 110 \mathrm{mg}, 150 \mathrm{mg}, 200 \mathrm{mg}, 265 \mathrm{mg}$, and $300 \mathrm{mg}$ doses once daily (QD), and $125 \mathrm{mg}$ and $150 \mathrm{mg}$ twice daily (BID) (Figure 2). Median treatment duration with formulation 1 was 32.5 days (range 2-269 days). Three patients experienced DLTs (one Grade 3 abnormal coagulation with sulfatinib $50 \mathrm{mg}$ QD; one Grade 3 upper gastrointestinal [GI] hemorrhage with sulfatinib $265 \mathrm{mg}$
QD; one Grade 3 abnormal hepatic function with sulfatinib $150 \mathrm{mg}$ BID). Twenty-three patients received sulfatinib formulation 2 during the dose-escalation phase at doses of $200 \mathrm{mg}, 300 \mathrm{mg}$, and $350 \mathrm{mg}$ QD (Figure 2). In addition, 11 patients were treated with $300 \mathrm{mg}$ or $350 \mathrm{mg}$ QD sulfatinib formulation 2 during the expansion phase. Median treatment duration with formulation 2 was 147.5 days (range 9-644 days). One patient receiving sulfatinib formulation 2 at 200 mg QD experienced a DLT (Grade 3 increased alanine transaminase [ALT]/aspartate aminotransferase [AST]).

MTD was not reached with sulfatinib doses of up to $350 \mathrm{mg}$ QD (formulation 2). The initial plan was to escalate the formulation 2 dose up to $400 \mathrm{mg}$ QD; however, drug exposure (AUC, area under the concentration-time curve; $\mathrm{C}_{\max }$, peak concentration) at $350 \mathrm{mg}$ QD was no higher than that at $300 \mathrm{mg}$ QD. Based on the available PK, safety, and efficacy data, the investigator and sponsor agreed there would be no further dose escalation even though MTD had not been reached.

\section{Safety}

Forty-two patients $(97.7 \%)$ receiving formulation 1 experienced one or more adverse events (AEs). The most common treatment-related AEs (TRAEs) (occurring in $\geq 10 \%$ patients) were: asthenia; increased blood bilirubin; protein present in urine; increased AST; diarrhea; increased blood pressure; hypomagnesemia; increased white blood cell count; abdominal pain; blood in urine; hypocalcemia; hypokalemia; and pyrexia. Grade 3 TRAE incidence was $25.6 \%$, with increased AST $(n=2,4.7 \%)$ and decreased hemoglobin $(\mathrm{n}=2,4.7 \%)$ most common. Other Grade 3 TRAEs (abdominal pain, diarrhea, upper GI hemorrhage, gastric dysfunction, abnormal hepatic function, gastroenteritis, infection, increased ALT, increased blood pressure, abnormal coagulation test, hypokalemia, hypoproteinemia, nephrotic syndrome, and pelvi-ureteric obstruction) each occurred in a single patient $(2.3 \%)$. There were no Grade 4 or 5 TRAEs. There were five serious AEs (SAEs) with formulation 1, three of which were considered by the investigator as possibly related to the study drug: a Grade 3 nephrotic syndrome and a Grade 3 upper GI hemorrhage, both in patients receiving $265 \mathrm{mg}$ QD; and a Grade 3 hepatic function abnormality in a patient receiving $150 \mathrm{mg}$ BID. These three patients discontinued sulfatinib and received supportive care. The patient with a Grade 3 hepatic function abnormality died 23 days after the last study dose; disease progression was considered the primary cause of death.

All patients treated with formulation 2 experienced at least one AE. The most common TRAEs of any grade (occurring in $\geq 10 \%$ of patients) are summarized in Table 3. Overall Grade 3 and 4 TRAE incidence was $47.1 \%$, and the most common was proteinuria (14.7\%; Table 3$)$. No Grade 5 AEs were reported. Eight SAEs were reported for formulation 2, two of which were considered by the investigator to be possibly related to the study drug: a Grade 3 upper GI hemorrhage in the $300 \mathrm{mg}$ QD dose 
Table 1: Sulfatinib kinase selectivity profile

\begin{tabular}{lc}
\hline Kinase & $\mathbf{I C}_{\mathbf{5 0}}(\boldsymbol{\mu M})$ \\
\hline VEGFR 1 & 0.002 \\
VEGFR 2 & 0.024 \\
VEGFR 3 & 0.001 \\
FGFR1 & 0.015 \\
CSF1R & 0.004 \\
TrkB & 0.041 \\
FLT3 & 0.067 \\
278 other kinases & $>0.150$ \\
\hline
\end{tabular}

FLT3: fms-related tyrosine kinase $3 ; \mathrm{IC}_{50}$ : half maximal inhibitory concentration; TrkB: tropomyosin receptor kinase $\mathrm{B}$.

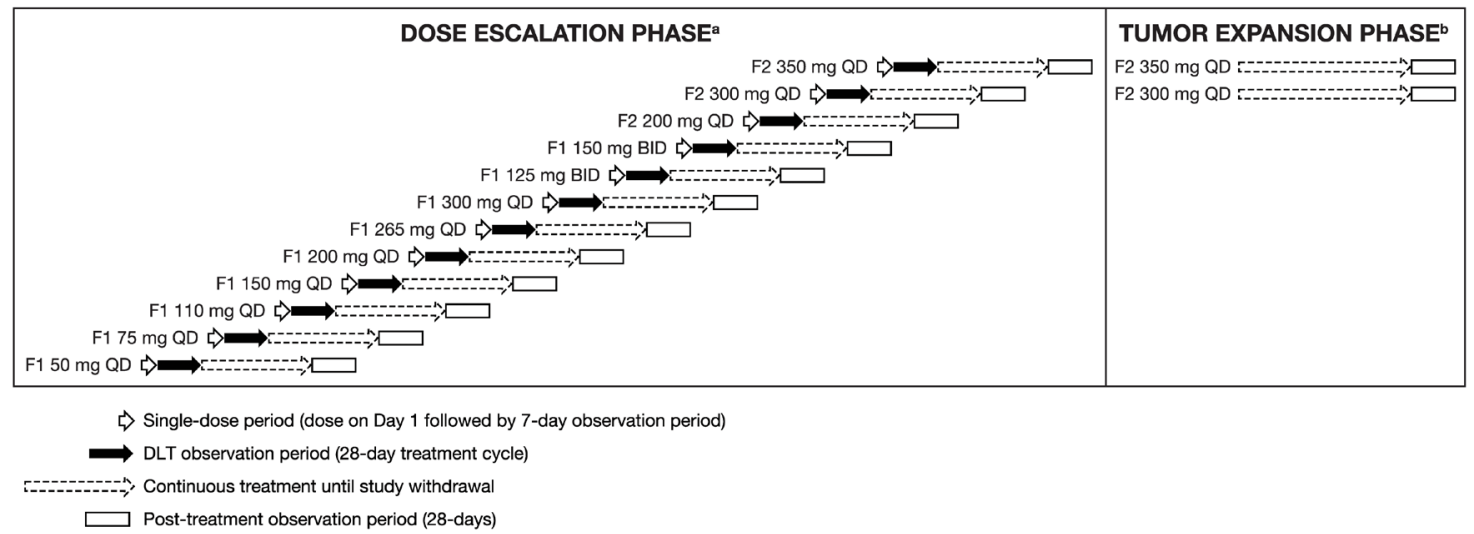

Figure 1: Study design. a Sulfatinib dose was escalated (until MTD was met) according to a modified Fibonacci $3+3$ protocol. Each patient received the assigned dose for the study duration. ${ }^{\mathrm{b}}$ The tumor expansion phase was initiated following determination of the recommended phase II dose based on the results of the dose-escalation phase.

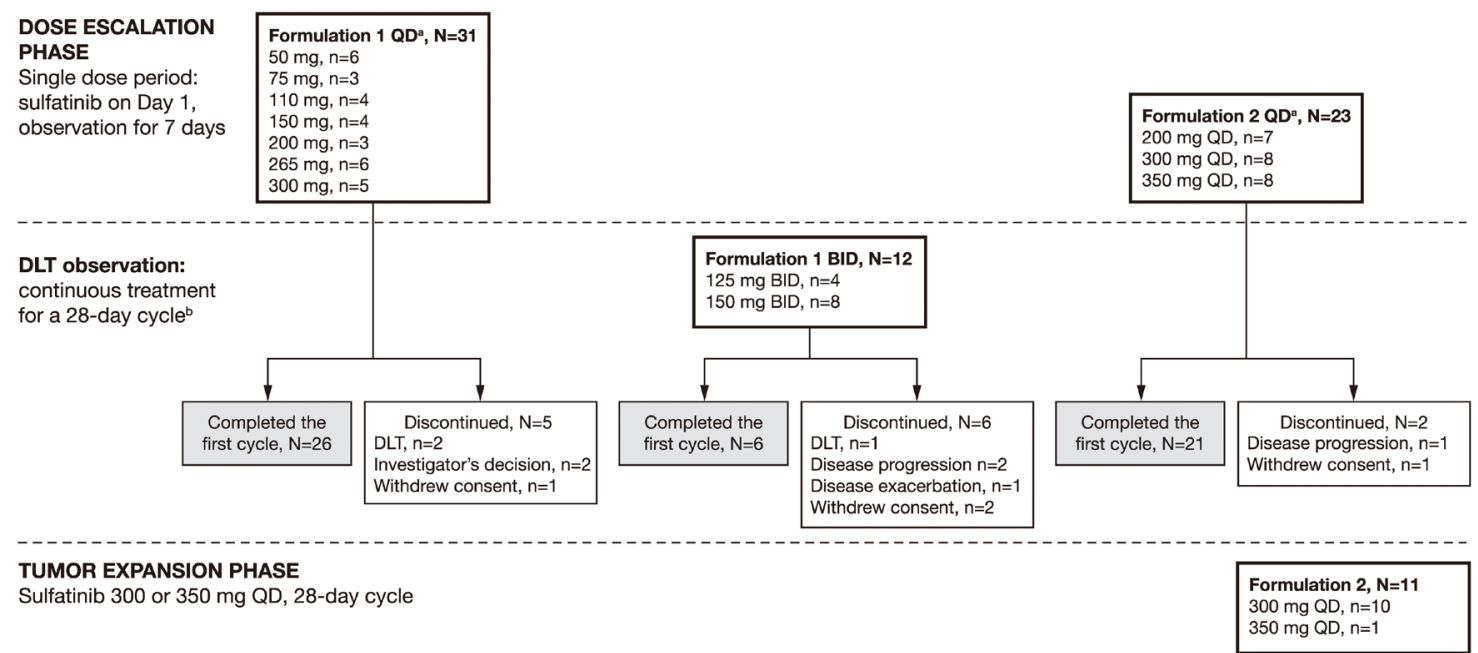

Figure 2: Patient configuration. a At enrolment, patients were assigned a dose sequentially according to the Fibonacci $3+3$ doseescalation design. Patients received that dose for the study duration. ${ }^{b}$ Patients who completed the DLT observation phase could remain on treatment at their original dose until disease progression or any other withdrawal criteria were met. 
Table 2: Patient baseline demographic and clinical characteristics

\begin{tabular}{|c|c|c|}
\hline Characteristic & $\begin{array}{l}\text { Formulation } 1 \\
\qquad(\mathrm{~N}=43)\end{array}$ & $\begin{array}{c}\text { Formulation } 2 \\
\qquad(\mathrm{~N}=\mathbf{3 4})\end{array}$ \\
\hline Median (range) age, years & $52.7(23.5-69.9)$ & $56.0(23.4-73.2)$ \\
\hline \multicolumn{3}{|l|}{ Gender, n (\%) } \\
\hline Male & $27(62.8)$ & $24(70.6)$ \\
\hline Female & $16(37.2)$ & $10(29.4)$ \\
\hline \multicolumn{3}{|l|}{ Tumor type, n (\%) } \\
\hline Colorectal carcinoma & $9(20.9)$ & 0 \\
\hline Hepatocellular carcinoma & $8(18.6)$ & $9(26.5)$ \\
\hline Stromal tumor & $8(18.6)$ & $1(2.9)$ \\
\hline NET $(\text { grade } 1 / 2)^{\mathrm{a}}$ & $7(16.3)$ & $21(61.8)$ \\
\hline Non-small cell lung cancer & $2(4.7)$ & 0 \\
\hline Renal cell carcinoma & $2(4.7)$ & 0 \\
\hline Other & $7(16.3)$ & $3(8.8)$ \\
\hline \multicolumn{3}{|l|}{ ECOG performance status, n (\%) } \\
\hline 0 & $10(23.3)$ & $4(11.8)$ \\
\hline 1 & $29(67.4)$ & $30(88.2)$ \\
\hline 2 & $4(9.3)$ & 0 \\
\hline Median (range) time since diagnosis, years & $1.9(0.1-11.2)$ & $0.8(0.0-6.8)$ \\
\hline \multicolumn{3}{|l|}{ Previous anti-tumor systemic therapy, n (\%) } \\
\hline Yes & $33(76.7)$ & $20(58.8)$ \\
\hline No & $10(23.3)$ & $14(41.2)$ \\
\hline
\end{tabular}

${ }^{a}$ NET pathology grading was categorized according to Ki67 index and tumor cell mitotic rate. Grade 1 and 2 tumors were also reported as well-differentiated NETs.

cohort; and a case of Grade 3 acute pancreatitis in the $350 \mathrm{mg}$ QD dose cohort. Most SAEs resolved, with the exception of an intra-abdominal hemorrhage (unrelated to the study drug) and an intervertebral disc protrusion (unlikely related to the study drug).

\section{Pharmacokinetic profile}

Sixty-eight patients were eligible for the steady-state PK assessment, including 40 patients who received sulfatinib formulation 1 and 28 who received formulation 2 (Table 4). For formulation 1, following QD administration for 14 days within the dose range of 50-265 mg, sulfatinib exposure (indicated by AUC) generally increased dose-proportionally. There was no AUC increase when the dose increased from $265 \mathrm{mg}$ to $300 \mathrm{mg}$. Median time to $\mathrm{C}_{\max }\left(\mathrm{T}_{\max }\right)$ ranged from 1.8 to 3.5 hours. Both $\mathrm{C}_{\max }$ and AUC showed high intersubject variability with coefficient of variation $(\mathrm{CV} \%)$ up to $69.5 \%$ for $\mathrm{C}_{\max }$ (75 mg group) and $68.8 \%$ for $\mathrm{AUC}(300$ mg group). Following BID administration for 14 days, mean AUC values were similar at 125 and $150 \mathrm{mg}$ (1977 versus $1952 \mathrm{ng} \cdot$ hour $/ \mathrm{mL}$ ) with a CV\% up to $64.8 \%$.
For formulation 2, following consecutive QD administration for 14 days, mean AUC at 200, 300, and $350 \mathrm{mg}$ was 4273,5116 , and $5289 \mathrm{ng} \cdot \mathrm{hour} / \mathrm{mL}$, respectively, indicating that sulfatinib exposure was similar at $300 \mathrm{mg}$ and $350 \mathrm{mg}$, but higher than that at 200 $\mathrm{mg}$. The inter-subject variability was high, as indicated by a CV\% of up to $55 \%$ for AUC and $73.1 \%$ for $\mathrm{C}_{\max }$. Median $\mathrm{T}_{\max }$ ranged from 1.0 to 2.0 hours for the test dose levels.

\section{Clinical response}

Among 43 patients treated with sulfatinib formulation 1, 12 patients were not evaluable for efficacy, either because they did not have a post-treatment tumor assessment, or because they exhibited SD at the first post-treatment assessment (week 4), but no additional assessment to demonstrate that the SD continued for a minimum of six weeks from baseline. Of the 31 patients evaluable by Response Evaluation Criteria in Solid Tumors (RECIST) Version 1.0, none achieved complete 
Table 3: TRAEs occurring in $\geq \mathbf{1 0 \%}$ of patients treated with sulfatinib formulation $2(\mathrm{~N}=34)$

\begin{tabular}{|c|c|c|c|c|c|c|c|c|}
\hline \multirow{2}{*}{$\begin{array}{l}\text { System organ class } \\
\text { Preferred term, n (\%) }\end{array}$} & \multicolumn{2}{|c|}{$200 \mathrm{mg} Q \mathrm{QD}, \mathrm{N}=7$} & \multicolumn{2}{|c|}{$300 \mathrm{mg} Q D, N=18$} & \multicolumn{2}{|c|}{$350 \mathrm{mg} Q \mathrm{QD}, \mathrm{N}=9$} & \multicolumn{2}{|c|}{ Total, $N=34$} \\
\hline & $\begin{array}{l}\text { Any } \\
\text { grade }\end{array}$ & $\begin{array}{c}\text { Grade } \\
3 / 4\end{array}$ & $\begin{array}{l}\text { Any } \\
\text { grade }\end{array}$ & $\begin{array}{c}\text { Grade } \\
3 / 4\end{array}$ & $\begin{array}{l}\text { Any } \\
\text { grade }\end{array}$ & $\begin{array}{c}\text { Grade } \\
3 / 4\end{array}$ & $\begin{array}{l}\text { Any } \\
\text { grade }\end{array}$ & $\begin{array}{c}\text { Grade } \\
3 / 4\end{array}$ \\
\hline \multicolumn{9}{|c|}{ Blood and lymphatic system disorders } \\
\hline Anemia & 0 & 0 & $2(11.1)$ & 0 & $2(22.2)$ & $1(11.1)$ & $4(11.8)$ & $1(2.9)$ \\
\hline \multicolumn{9}{|l|}{ Cardiac disorders } \\
\hline Sinus bradycardia & 0 & 0 & $4(22.2)$ & 0 & $1(11.1)$ & 0 & $5(14.7)$ & 0 \\
\hline \multicolumn{9}{|l|}{ Gastrointestinal disorders } \\
\hline Diarrhea & $3(42.9)$ & 0 & $8(44.4)$ & $1(5.6)$ & $8(88.9)$ & $1(11.1)$ & $19(55.9)$ & $2(5.9)$ \\
\hline Abdominal discomfort & $2(28.6)$ & 0 & $3(16.7)$ & 0 & $6(66.7)$ & 0 & $11(32.4)$ & 0 \\
\hline Nausea & $3(42.9)$ & 0 & $4(22.2)$ & 0 & $2(22.2)$ & 0 & $9(26.5)$ & 0 \\
\hline Abdominal distention & $1(14.3)$ & 0 & $4(22.2)$ & 0 & $4(44.4)$ & 0 & $9(26.5)$ & 0 \\
\hline
\end{tabular}

\section{General disorders and administration site conditions}

\begin{tabular}{|c|c|c|c|c|c|c|c|c|}
\hline Asthenia & $2(28.6)$ & 0 & $4(22.2)$ & 0 & $6(66.7)$ & $1(11.1)$ & $12(35.3)$ & $1(2.9)$ \\
\hline Face edema & 0 & 0 & $2(11.1)$ & $1(5.6)$ & $5(55.6)$ & 0 & $7(20.6)$ & $1(2.9)$ \\
\hline Edema peripheral & 0 & 0 & $2(11.1)$ & $1(5.6)$ & $3(33.3)$ & 0 & $5(14.7)$ & $1(2.9)$ \\
\hline \multicolumn{9}{|l|}{ nvestigations } \\
\hline Blood pressure increased & 0 & 0 & $10(55.6)$ & $2(11.1)$ & $4(44.4)$ & 0 & $14(41.2)$ & $2(5.9)$ \\
\hline Blood TSH increased & $1(14.3)$ & 0 & $7(38.9)$ & 0 & $5(55.6)$ & 0 & $13(38.2)$ & 0 \\
\hline Blood bilirubin increased & $2(28.6)$ & 0 & $8(44.4)$ & $1(5.6)$ & $3(33.3)$ & 0 & $13(38.2)$ & $1(2.9)$ \\
\hline AST increased & $2(28.6)$ & $\begin{array}{c}1 \\
(14.3)\end{array}$ & $6(33.3)$ & $1(5.6)$ & $4(44.4)$ & 0 & $12(35.3)$ & $2(5.9)$ \\
\hline Blood triglycerides increased & 0 & 0 & $5(27.8)$ & 0 & $7(77.8)$ & 0 & $12(35.3)$ & 0 \\
\hline WBC count decreased & $1(14.3)$ & 0 & $5(27.8)$ & 0 & $5(55.6)$ & 0 & $11(32.4)$ & 0 \\
\hline Neutrophil count decreased & $2(28.6)$ & 0 & $4(22.2)$ & $1(5.6)$ & $4(44.4)$ & 0 & $10(29.4)$ & $1(2.9)$ \\
\hline Blood albumin decreased & $1(14.3)$ & 0 & $8(44.4)$ & 0 & $1(11.1)$ & 0 & $10(29.4)$ & 0 \\
\hline Platelet count decreased & $2(28.6)$ & 0 & $3(16.7)$ & 0 & $5(55.6)$ & $2(22.2)$ & $10(29.4)$ & $2(5.9)$ \\
\hline Abnormal ECG T-wave & $1(14.3)$ & 0 & $6(33.3)$ & 0 & $2(22.2)$ & 0 & $9(26.5)$ & 0 \\
\hline Blood uric acid increased & $1(14.3)$ & 0 & $4(22.2)$ & $1(5.6)$ & $4(44.4)$ & 0 & $9(26.5)$ & $1(2.9)$ \\
\hline ALT increased & $2(28.6)$ & $\begin{array}{c}1 \\
(14.3)\end{array}$ & $2(11.1)$ & 0 & $2(22.2)$ & 0 & $6(17.6)$ & $1(2.9)$ \\
\hline Blood creatinine increased & $1(14.3)$ & 0 & 0 & 0 & $5(55.6)$ & 0 & $6(17.6)$ & 0 \\
\hline Thyroxine free decreased & 0 & 0 & $5(27.8)$ & 0 & $1(11.1)$ & 0 & $6(17.6)$ & 0 \\
\hline Hemoglobin decreased & $1(14.3)$ & 0 & $1(5.6)$ & $1(5.6)$ & $3(33.3)$ & $1(11.1)$ & $5(14.7)$ & $2(5.9)$ \\
\hline Thyroid function test abnormal & $1(14.3)$ & 0 & $1(5.6)$ & 0 & $2(22.2)$ & 0 & $4(11.8)$ & 0 \\
\hline \multicolumn{9}{|c|}{ Ietabolism and nutrition disorders } \\
\hline Hypoproteinemia & 0 & 0 & $10(55.6)$ & 0 & $7(77.8)$ & 0 & $17(50.0)$ & 0 \\
\hline Hypocalcemia & 0 & 0 & $7(38.9)$ & 0 & $5(55.6)$ & 0 & $12(35.3)$ & 0 \\
\hline
\end{tabular}




\begin{tabular}{|c|c|c|c|c|c|c|c|c|}
\hline \multirow{2}{*}{$\begin{array}{l}\text { System organ class } \\
\text { Preferred term, n (\%) }\end{array}$} & \multicolumn{2}{|c|}{200 mg QD, N=7 } & \multicolumn{2}{|c|}{$300 \mathrm{mg} Q \mathrm{QD}, \mathrm{N}=18$} & \multicolumn{2}{|c|}{$350 \mathrm{mg}$ QD, N=9 } & \multicolumn{2}{|c|}{ Total, $\mathbf{N}=34$} \\
\hline & $\begin{array}{c}\text { Any } \\
\text { grade }\end{array}$ & $\begin{array}{c}\text { Grade } \\
3 / 4\end{array}$ & $\begin{array}{c}\text { Any } \\
\text { grade }\end{array}$ & $\begin{array}{c}\text { Grade } \\
3 / 4\end{array}$ & $\begin{array}{c}\text { Any } \\
\text { grade }\end{array}$ & $\begin{array}{c}\text { Grade } \\
3 / 4\end{array}$ & $\begin{array}{c}\text { Any } \\
\text { grade }\end{array}$ & $\begin{array}{c}\text { Grade } \\
3 / 4\end{array}$ \\
\hline Decreased appetite & 0 & 0 & $6(33.3)$ & 0 & $5(55.6)$ & 0 & $11(32.4)$ & 0 \\
\hline Hypokalemia & $2(28.6)$ & 0 & $4(22.2)$ & 0 & $2(22.2)$ & $1(11.1)$ & $8(23.5)$ & $1(2.9)$ \\
\hline Hypertriglyceridemia & 0 & 0 & $4(22.2)$ & 0 & $4(44.4)$ & 0 & $8(23.5)$ & 0 \\
\hline Hyperuricemia & 0 & 0 & $4(22.2)$ & $1(5.6)$ & $3(33.3)$ & 0 & $7(20.6)$ & $1(2.9)$ \\
\hline Hypophosphatemia & $2(28.6)$ & 0 & $3(16.7)$ & $2(11.1)$ & $1(11.1)$ & 0 & $6(17.6)$ & $2(5.9)$ \\
\hline Hyponatremia & 0 & 0 & $3(16.7)$ & 0 & $1(11.1)$ & 0 & $4(11.8)$ & 0 \\
\hline Hypercholesterolemia & 0 & 0 & 0 & 0 & $4(44.4)$ & 0 & $4(11.8)$ & 0 \\
\hline \multicolumn{9}{|c|}{ Musculoskeletal and connective tissue disorders } \\
\hline Back pain & $1(14.3)$ & 0 & $3(16.7)$ & 0 & $1(11.1)$ & 0 & $5(14.7)$ & 0 \\
\hline \multicolumn{9}{|l|}{ Nervous system disorders } \\
\hline Dizziness & $1(14.3)$ & 0 & $2(11.1)$ & 0 & $3(33.3)$ & 0 & $6(17.6)$ & 0 \\
\hline \multicolumn{9}{|c|}{ Renal and urinary disorders } \\
\hline Proteinuria & $2(28.6)$ & 0 & $11(61.1)$ & $3(16.7)$ & $7(77.8)$ & $2(22.2)$ & $20(58.8)$ & $5(14.7)$ \\
\hline \multicolumn{9}{|l|}{ Vascular disorders } \\
\hline Hypertension & 0 & 0 & $5(27.8)$ & $1(5.6)$ & $2(22.2)$ & 0 & $7(20.6)$ & $1(2.9)$ \\
\hline
\end{tabular}

TRAE: treatment-related adverse event; TSH: thyroid stimulating hormone; WBC: white blood cell; ECG: electrocardiogram.

response (CR) or partial response (PR). Eight patients had stable disease (SD) and 23 had progressive disease (PD).

Among 34 patients treated with formulation 2 , six were not evaluable for response due to early discontinuation without adequate post-treatment tumor evaluation. Of the 28 patients evaluable by RECIST criteria, nine achieved PR (Figure 3), including one patient with hepatocellular carcinoma receiving sulfatinib 200 $\mathrm{mg} \mathrm{QD}$, and eight with neuroendocrine tumors (NETs) receiving sulfatinib 300 or $350 \mathrm{mg}$ QD. There were 15 patients with SD (10 with NETs, three with hepatocellular carcinoma, one with GI stromal tumors, and one with an abdominal malignancy) and four patients with PD.

The objective response rate (ORR) for all 77 patients was $11.7 \%(9 / 77)$ and disease control rate (DCR) was $41.6 \%(32 / 77)$. The ORR of patients treated with sulfatinib formulation 2 was $26.5 \%(9 / 34)$ and DCR was $70.6 \%$ (24/34). Tumor response rate was similar among patients receiving higher formulation 2 doses. In the 300 mg QD cohort, ORR was $27.8 \%(5 / 18)$ and DCR was $66.7 \%(12 / 18)$, while in the $350 \mathrm{mg}$ QD cohort, ORR was $33.3 \%$ (3/9) and DCR was $77.8 \%$ (7/9).

There were 21 patients with well-differentiated NETs (grade 1 or 2) treated with sulfatinib formulation 2 at 200-350 mg QD. Within this subgroup, eight patients achieved PR, 10 achieved SD, and three were not evaluable for response, with an ORR of $38.1 \%$ $(8 / 21)$ and DCR of $85.7 \%(18 / 21)$. Tumor origins of the eight NET patients who achieved PR were: pancreas $(n=3)$; duodenum $(n=1)$; rectum $(n=1)$; thymus $(n=1)$; and unknown $(\mathrm{n}=2)$. Median time to response (TTR) was 3.0 months (range 1.3-10.2 months). Median duration of response (DoR) was 15.7 months (95\% confidence interval [CI]: 3.8-not reached [NR]). Median progression free survival (PFS) was 16.9 months (95\% CI: 9.5-NR) (Figure 4). Notably, three NET patients who had previously been treated, but progressed on VEGFR kinase inhibitors (such as sunitinib or famitinib), obtained clinical benefit from sulfatinib, with two patients achieving SD and one achieving PR (treatment duration from 5.1 to 11.6 months).

\section{DISCUSSION}

Sulfatinib, a potent oral kinase inhibitor targeting VEGFR (1, 2, 3), FGFR1, and CSF1R with good selectivity, has demonstrated anti-angiogenic and anti-tumor activity in preclinical studies (Hutchison MediPharma, unpublished data). This first-in-human, phase I study investigated the safety, PK characteristics, 
Table 4: Sulfatinib pharmacokinetic parameters on day 14 of continuous dosing

\begin{tabular}{|c|c|c|c|c|c|c|c|c|c|}
\hline \multirow[b]{2}{*}{$\begin{array}{l}\text { PK } \\
\text { parameter }\end{array}$} & \multicolumn{9}{|c|}{ Sulfatinib formulation 1 dose } \\
\hline & $\begin{array}{c}50 \mathrm{mg} \\
\mathrm{QD} \\
(\mathrm{N}=\mathbf{5})\end{array}$ & $\begin{array}{c}75 \mathrm{mg} \\
\mathrm{QD} \\
(\mathrm{N}=3)\end{array}$ & $\begin{array}{c}110 \mathrm{mg} \\
\text { QD } \\
(\mathrm{N}=4)\end{array}$ & $\begin{array}{c}150 \mathrm{mg} \\
\text { QD } \\
(\mathrm{N}=3)\end{array}$ & $\begin{array}{c}200 \mathrm{mg} \text { QD } \\
(\mathrm{N}=3)\end{array}$ & $\begin{array}{c}265 \mathrm{mg} \\
\text { QD } \\
(\mathrm{N}=6)\end{array}$ & $\begin{array}{c}300 \text { mg QD } \\
(\mathrm{N}=5)\end{array}$ & $\begin{array}{c}125 \mathrm{mg} \\
\text { BID } \\
(\mathrm{N}=3)\end{array}$ & $\begin{array}{c}150 \mathrm{mg} \\
\mathrm{BID} \\
(\mathrm{N}=\mathbf{8})\end{array}$ \\
\hline $\begin{array}{l}\text { Mean } \mathrm{C}_{\max } \\
(\mathrm{CV} \%), \mathrm{ng} / \\
\mathrm{mL}\end{array}$ & $\begin{array}{c}84 \\
(56.4)\end{array}$ & $\begin{array}{c}123 \\
(69.5)\end{array}$ & $\begin{array}{c}262 \\
(35.3)\end{array}$ & $\begin{array}{c}293 \\
(35.7)\end{array}$ & $\begin{array}{c}370 \\
(20.3)\end{array}$ & $\begin{array}{c}498 \\
(66.9)\end{array}$ & $\begin{array}{c}546 \\
(67.3)\end{array}$ & $\begin{array}{c}267 \\
(52.1)\end{array}$ & $\begin{array}{c}248 \\
(65.9)\end{array}$ \\
\hline $\begin{array}{l}\text { Median } \mathrm{T}_{\max } \\
\text { (range), } \\
\text { hour }\end{array}$ & $\begin{array}{c}1.8 \\
(1.0-4.0)\end{array}$ & $\begin{array}{c}2.0 \\
(1.0-4.0)\end{array}$ & $\begin{array}{c}1.5 \\
(0-4.0)\end{array}$ & $\begin{array}{c}1.0 \\
(1.0-1.0)\end{array}$ & $\begin{array}{c}3.0 \\
(1.0-4.0)\end{array}$ & $\begin{array}{c}3.5 \\
(1.0-4.0)\end{array}$ & $\begin{array}{c}2.8 \\
(1.0-4.0)\end{array}$ & $\begin{array}{c}3.0 \\
(1.0-4.0)\end{array}$ & $\begin{array}{c}3.4 \\
(1.0-8.0)\end{array}$ \\
\hline $\begin{array}{l}\text { Mean AUC } \\
(\mathrm{CV} \%), \\
\mathrm{ng} \cdot \text { hour } / \mathrm{mL}\end{array}$ & $\begin{array}{c}654 \\
(51.2)\end{array}$ & $\begin{array}{c}964 \\
(32.3)\end{array}$ & $\begin{array}{l}2579 \\
(28.2)\end{array}$ & $2308(48.8)$ & $\begin{array}{l}3314 \\
(13.1)\end{array}$ & $\begin{array}{c}5958 \\
(65.2)\end{array}$ & $\begin{array}{l}5403 \\
(68.8)\end{array}$ & $\begin{array}{c}1977 \\
(59.8)\end{array}$ & $\begin{array}{l}1952 \\
(64.8)\end{array}$ \\
\hline \multirow[t]{2}{*}{$\begin{array}{l}\text { PK } \\
\text { parameter }\end{array}$} & \multicolumn{9}{|c|}{ Sulfatinib formulation 2 dose } \\
\hline & & $\begin{array}{c}200 \mathrm{mg} \text { QD } \\
\quad(\mathrm{N}=6)\end{array}$ & & & $\begin{array}{c}300 \mathrm{mg} Q D \\
(\mathrm{~N}=14)\end{array}$ & & & $\begin{array}{c}350 \mathrm{mg} \text { QD } \\
\quad(\mathrm{N}=8)\end{array}$ & \\
\hline $\begin{array}{l}\text { Mean } \mathrm{C}_{\max } \\
(\mathrm{CV} \%), \mathrm{ng} / \\
\mathrm{mL}\end{array}$ & & $\begin{array}{c}549 \\
(73.1)\end{array}$ & & & $\begin{array}{c}625 \\
(54.6)\end{array}$ & & & $\begin{array}{c}655 \\
(32.5)\end{array}$ & \\
\hline $\begin{array}{l}\text { Median } \mathrm{T}_{\max } \\
\text { (range), } \\
\text { hour }\end{array}$ & & $\begin{array}{c}1.0 \\
(1.0-2.0)\end{array}$ & & & $\begin{array}{c}2.0 \\
(1.0-4.1)\end{array}$ & & & $\begin{array}{c}2.0 \\
(2.0-4.0)\end{array}$ & \\
\hline $\begin{array}{l}\text { Mean AUC } \\
(\mathrm{CV} \%), \\
\mathrm{ng} \cdot \text { hour/mL }\end{array}$ & & $\begin{array}{l}4273 \\
(55.0)\end{array}$ & & & $\begin{array}{l}5116 \\
(50.4)\end{array}$ & & & $\begin{array}{l}5289 \\
(37.6)\end{array}$ & \\
\hline
\end{tabular}

${ }^{\mathrm{a}} \mathrm{AUC}_{0-24}$ for $\mathrm{QD}$ and $\mathrm{AUC}_{0-12}$ for BID.

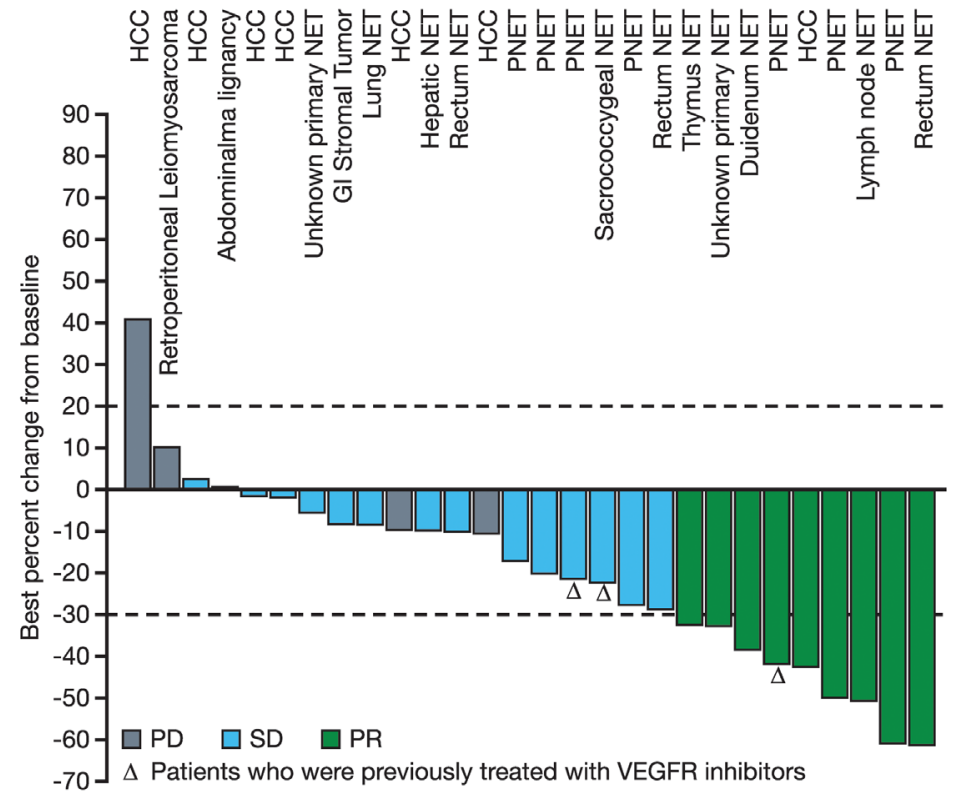

Figure 3: Best percent change in tumor size (sum of diameter of target lesions) compared with baseline for efficacyevaluable patients treated with sulfatinib formulation $2(\mathbf{N}=\mathbf{2 8})$. PNET: pancreatic neuroendocrine tumor. 
and preliminary anti-tumor activity of sulfatinib in patients with advanced solid tumors.

MTD was not reached within sulfatinib doses of 50$350 \mathrm{mg}$ QD and 125-150 mg BID, and sulfatinib appeared to be generally well tolerated. Most AEs were mild to moderate and could be managed through dose adjustment or supportive care. The most commonly reported AEs, including proteinuria, hypertension, and diarrhea, were consistent with AEs seen with VEGFR tyrosine kinase inhibitors [9-12].

Sulfatinib demonstrated promising anti-tumor activity against advanced solid tumors in our study. Nine out of the 77 patients had a confirmed PR and 23 had sustainable SD. Clinical efficacy was observed with sulfatinib formulation 2 at doses from $200 \mathrm{mg}$ QD, with nine patients achieving a PR and 15 achieving SD. PK analyses demonstrated that sulfatinib was rapidly absorbed and drug exposure (AUC and $\mathrm{C}_{\max }$ ) generally increased with dose escalation. Drug exposure began to plateau at a dose of $265 \mathrm{mg}$ for formulation 1 and $300 \mathrm{mg}$ for formulation 2, suggesting potential absorption saturation. Inter-patient variation in drug exposure was moderate to high across all dose levels for formulation 1, and appeared moderately improved for formulation 2 .

Sulfatinib was well tolerated up to $350 \mathrm{mg}$ QD. Drug exposure (AUC) did not increase with a dose increase from 300 to $350 \mathrm{mg}$ QD (formulation 2). This finding suggested potential absorption saturation and that further dose escalation would not achieve an increase in drug exposure. However, there was a higher incidence of Grade $\geq 3$ AEs with the $350 \mathrm{mg}$ dose compared to 300 $\mathrm{mg}$ during the first cycle of the continuous dose period. Although sample size was small, the two dose cohorts demonstrated comparable anti-tumor activity. Together, the PK, safety, and efficacy data support the selection of $300 \mathrm{mg}$ QD as the recommended phase II dose.

Unresectable or metastatic NET is a rare and lifethreatening disease with limited treatment options [1315]. Median survival varies from several months to a few years depending on primary tumor site [16]. In recent years, only two targeted therapies have been approved by the United States Food and Drug Administration for treatment of advanced well-differentiated NETs: sunitinib, a multi-kinase inhibitor mainly targeting angiogenesis, and everolimus, an oral mammalian target of rapamycin (mTOR) inhibitor. In a phase III sunitinib trial in pancreatic NET patients, ORR was $9.3 \%$ with PFS of 11.0 months [11], although sunitinib failed to demonstrate effectiveness in extra-pancreatic NET patients. In phase III trials, median PFS of pancreatic NET or GI and lung NET patients treated with everolimus was both 11.0 months, while ORR was only $5 \%$ and $2 \%$, respectively $[17,18]$.

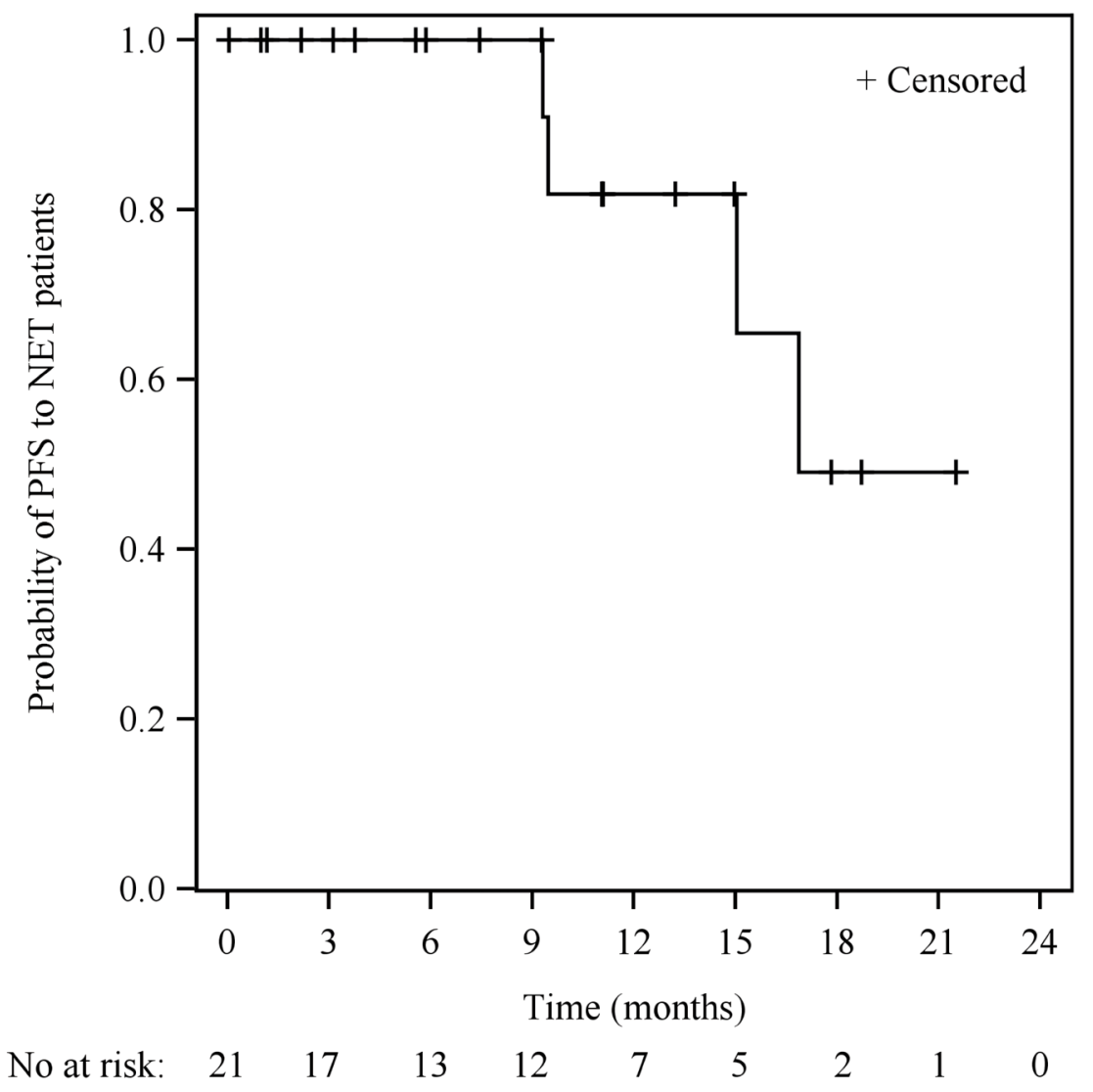

Figure 4: Kaplan-Meier survival curve of PFS in NET patients treated with sulfatinib formulation 2 (N=21). 
The present study included 21 patients with advanced NETs treated with sulfatinib formulation 2 . Robust clinical activity was demonstrated in these cases, with an ORR of $38.1 \%$, DCR of $85.7 \%$, and a median PFS of 16.9 months (95\% CI: 9.5-NR). Notably, anti-tumor activity was demonstrated by sulfatinib in NET patients regardless of tumor origin, and also in three patients who had previously failed prior VEGFR inhibitor treatment. This suggests that sulfatinib, which simultaneously targets tumor angiogenesis and immune evasion, may provide clinical benefit for NET patients [7, 19]. The efficacy analysis should be interpreted with caution due to the small sample size, and the non-randomized, openlabel study design with no comparator. Nevertheless, the preliminary results of this study provide sufficient support to warrant further sulfatinib anti-tumor efficacy evaluation. Investigations into the sulfatinib mechanism of anti-tumor activity are ongoing, both in preclinical and clinical settings, and may provide more support for the use of sulfatinib in advanced solid tumors.

In summary, sulfatinib has demonstrated promising anti-tumor activity in patients with advanced solid tumors, especially NETs, and was generally well tolerated. Our results will be further validated in a phase Ib/II study in NET patients (NCT02267967) and in two randomized, double-blind, placebo-controlled, multicenter phase III trials: one in patients with pancreatic NETs (NCT02589821) and one in patients with extra-pancreatic NET (NCT02588170).

\section{MATERIALS AND METHODS}

\section{Patients}

Patients were recruited from the Affiliated Hospital Cancer Center (the 307th Hospital of Chinese People's Liberation Army), Academy of Military Medical Sciences, Beijing, China, and Peking University Cancer Hospital, Beijing, China. Patients with recurrent and/or metastatic malignant solid tumors were eligible for this study if they had shown disease progression after standard therapy or were unable to receive standard therapy. Eligible patients were 18-75 years old, with an Eastern Cooperative Oncology Group (ECOG) performance status $\leq 2$, and a life expectancy of $>3$ months. Patients with uncontrolled brain metastases were excluded. Pre-treatment evaluations included: a physical examination; ECOG performance status; laboratory tests for renal, liver, and metabolic functions; cardiac function (electrocardiogram and ultrasonic cardiogram); and a pregnancy test for female patients of childbearing age.

\section{Study design and dose administration}

The primary objectives of this open-label, first-inhuman phase I study (NCT02133157) were to determine
MTD and the phase II dose of sulfatinib, and to evaluate the safety of sulfatinib in patients with advanced solid tumors. The secondary and exploratory objectives included evaluation of sulfatinib PK and preliminary antitumor activity. The study consisted of a dose-escalation phase (split into a single-dose period and continuousdose period) and a tumor-specific expansion phase. Two sulfatinib formulations were used during the study: formulation $1(5,25$, and $50 \mathrm{mg}$ capsules) and formulation 2 (50 and $200 \mathrm{mg}$ capsules). The study was conducted in accordance with the Good Clinical Practice Guidelines of the International Council for Harmonization of Technical Requirements for Pharmaceuticals for Human Use. The protocol was approved by each participating institution's ethics review board. All patients provided written informed consent.

During the dose-escalation phase, patients were given a single dose of sulfatinib and monitored for AEs for seven days. If no clinically significant toxicities were observed, patients could enter the 28-day DLT observation phase where they received sulfatinib continuously for 28 days. DLTs were assessed at the end of the 28-day period. If no patients experienced a DLT during the 28day period, the dose was escalated. After completion of the DLT observation phase, patients could continue treatment at their current dose (if they were judged by the investigator to be benefiting from treatment) until any of the withdrawal criteria (investigator's decision that withdrawal was in the patient's best interest, intolerable toxicity, disease progression) were met.

The study used a modified Fibonacci $3+3$ doseescalation design with at least three evaluable patients treated with each dose. The MTD was defined as the maximum dose at which no more than one of six evaluable patients experienced a DLT during the first 28-day treatment period (cycle). For each dose, if no patients experienced a DLT during the treatment cycle, the dose was escalated for the next dose cohort. If one of the first three patients treated at a dose experienced a DLT, three additional patients were added to expand the cohort. If two or more of the first three or overall six patients experienced a DLT, the MTD was considered exceeded and the previous lower dose would be reassessed to determine the MTD. Patients who completed the first treatment cycle and fulfilled $\geq 75 \%$ of the planned accumulating dose, or who experienced a DLT any time during the first treatment cycle, were considered as DLTevaluable patients. Dose reductions were not permitted in the first treatment cycle.

After the MTD and recommended phase II dose were established and preliminary efficacy data from the dose-escalation phase had demonstrated an effective dose range (a PR had been observed), the study was expanded to investigate tumor response at the identified doses (300 $\mathrm{mg}$ and $350 \mathrm{mg}$ QD [formulation 2]) in patients with advanced solid tumors. Patients with NETs were enrolled 
with priority as preliminary efficacy was shown in these tumor types in the dose-escalation stage.

\section{Endpoints and analyses}

Safety and tolerability were assessed in all patients who received at least one dose of sulfatinib throughout the study. AEs were recorded throughout the study. All AEs were coded by organ system using preferred terms as per the Medical Dictionary for Regulatory Activities (MedDRA) Version 17.0, and were graded using the National Cancer Institute Common Terminology Criteria for Adverse Events (NCI CTCAE) Version 3.0. AE frequency, severity, and relationship to study drug were summarized and tabulated, together with SAEs or deaths. TRAEs were defined as AEs that were considered by the investigators to be possibly, probably or definitely related to the study treatment.

DLTs were defined as any of the following toxicities occurring in the first continuous-dosing treatment cycle (day 1-28) of the dose-escalation phase: any nonhematologic toxicity $\geq$ Grade 3 in severity, except for fatigue, nausea, vomiting, diarrhea, constipation, pain, and hypertension, which were considered DLTs if they were $\geq$ Grade 3 after adequate treatment; hematologic toxicities, including Grade 4 decreases in white blood cell count, platelet count or hemoglobin; Grade 3 febrile neutropenia; and Grade 3 decreases in platelets with hemorrhage tendency. Physical examinations, ECOG performance status, and laboratory tests were obtained in the single-dose screening period at day 1 , and during the continuous-dose period were collected weekly in the first treatment cycle, every two weeks in the second treatment cycle, and every four weeks in the third treatment cycle onwards.

For the assessment of sulfatinib PK at steady state, plasma samples were collected from each patient prior to treatment, and at $1,2,4,8,12$, and 24 hours on day 14 for QD cohorts or at $1,4,8$, and 12 hours following the first dose on day 14 for BID cohorts. PK parameters analyzed were $\mathrm{AUC}, \mathrm{C}_{\max }$, and $\mathrm{T}_{\max }$. Phoenix WinNonlin 6.3 software was used to analyze descriptive statistics of concentration data and PK parameters, and to plot plasma concentrationtime curves. AUC was calculated using the linear trapezoidal area method.

Tumor response (an exploratory endpoint) was assessed according to RECIST Version 1.0 and measured at baseline, at the end of every treatment cycle in the first four cycles, and every other cycle thereafter. Patients with an initial assessment of CR or PR had this result confirmed by a repeat tumor assessment at least four weeks later. Calculations of the following parameters were made: ORR $(\mathrm{CR}+\mathrm{PR})$; SD defined as $\geq 1$ assessment of SD at least six weeks after study entry; DCR $(C R+P R+S D)$. TTR, DoR and PFS were analyzed in the subgroup of patients with NET.

\section{ACKNOWLEDGMENTS}

The authors thank the patients and their families for participation in this study. The authors also thank the nurses, clinical research coordinators, and GCP office staff at the Affiliated Hospital Cancer Center (the 307th Hospital of Chinese People's Liberation Army), Academy of Military Medical Sciences and Peking University Cancer Hospital. The authors thank Katie White, PhD, of Complete Medical Communications (funded by Hutchison MediPharma Limited) for editorial revisions.

\section{CONFLICTS OF INTEREST}

J.M. $\mathrm{Xu}$ is a consultant/advisory board member for Hutchison MediPharma Limited and has received speaker's bureau honoraria from Roche, Novartis, Merck, and Sanofi. L. Shen has received research funding from Taiho Pharma, Hengrui, and Roche; is a consultant member of Taiho Pharma, Hengrui, and Merck; and has received honoraria from Novartis, Merck, Roche, Prifzer, Sanofi, Taiho Pharma, and Hengrui. Jie L has received speaker honoraria from Novartis. C.R. Tan, Jing Li, C. Qi, K. Li, J. Wang, F. Zhou, Y. Hua, Y. Sai, Y.X. Ren, W.G. Qing, H. Jia, and W.G. Su are employees of Hutchison MediPharma Limited. Y. Wang, Y.L. Chen, R. Jia, and J.F. Gong have no conflicts of interest.

\section{GRANT SUPPORT}

This study was funded by Hutchison MediPharma Limited.

\section{REFERENCES}

1. Cébe-Suarez S, Zehnder-Fjällman A, Ballmer-Hofer K. The role of VEGF receptors in angiogenesis; complex partnerships. Cell Mol Life Sci. 2006; 63: 601-615.

2. Daniele G, Corral J, Molife LR, de Bono JS. FGF "Receptor inhibitors: role in cancer therapy. Curr Oncol Rep. 2012; 14: 111-119.

3. Folkman J. Role of angiogenesis in tumor growth and metastasis. Semin Oncol. 2002; 29: 15-18.

4. Ellis LM, Hicklin DJ. Pathways mediating resistance to vascular endothelial growth factor-targeted therapy. Clin Cancer Res. 2008; 14: 6371-6375.

5. Sitohy B, Nagy JA, Dvorak HF. Anti-VEGF/VEGFR therapy for cancer: reassessing the target. Cancer Res. 2012; 72: 1909-1914.

6. Voron T, Colussi O, Marcheteau E, Pernot S, Nizard M, Pointet AL, Latreche S, Bergaya S, Benhamouda N, Tanchot C, Stockmann C, Combe P, Berger A, et al. VEGF-A modulates expression of inhibitory checkpoints on CD8(+) T cells in tumors. J Exp Med. 2015; 212: 139-148. 
7. Katoh M. FGFR inhibitors: effects on cancer cells, tumor microenvironment and whole-body homeostasis (Review). Int J Mol Med. 2016; 38: 3-15.

8. Hutchison Medipharma Limited. Sulfatinib chemical structure and synthesis: US Patent 8,946,249 B2. 2015.

9. Motzer RJ, Hutson TE, Tomczak P, Michaelson MD, Bukowski RM, Rixe O, Oudard S, Negrier S, Szczylik C, Kim ST, Chen I, Bycott PW, Baum CM, et al. Sunitinib versus interferon alfa in metastatic renal-cell carcinoma. N Engl J Med. 2007; 356: 115-124.

10. Escudier B, Eisen T, Stadler WM, Szczylik C, Oudard S, Siebels M, Negrier S, Chevreau C, Solska E, Desai AA, Rolland F, Demkow T, Hutson TE, et al. Sorafenib in advanced clear-cell renal-cell carcinoma. N Engl J Med. 2007; 356: 125-134.

11. Raymond E, Dahan L, Raoul JL, Bang YJ, Borbath I, Lombard-Bohas C, Valle J, Metrakos P, Smith D, Vinik A, Chen JS, Hörsch D, Hammel P, et al. Sunitinib malate for the treatment of pancreatic neuroendocrine tumors. N Engl J Med. 2011; 364: 501-513.

12. Zhou A, Zhang W, Chang C, Chen X, Zhong D, Qin Q, Lou D, Jiang H, Wang J. Phase I study of the safety, pharmacokinetics and antitumor activity of famitinib. Cancer Chemother Pharmacol. 2013; 72: 1043-1053.

13. Öberg K, Knigge U, Kwekkeboom D, Perren A; ESMO Guidelines Working Group. Neuroendocrine gastro-enteropancreatic tumors: ESMO Clinical Practice Guidelines for diagnosis, treatment and follow-up. Ann Oncol. 2012; 23: vii124-vii130.

14. Öberg K, Hellman P, Ferolla P, Papotti M; ESMO Guidelines Working Group. Neuroendocrine bronchial and thymic tumors: ESMO Clinical Practice Guidelines for diagnosis, treatment and follow-up. Ann Oncol. 2012; 23: vii120-vii123.

15. Wong MH, Chan DL, Lee A, Li BT, Lumba S, Clarke SJ, Samra J, Pavlakis N. Systematic review and meta-analysis on the role of chemotherapy in advanced and metastatic neuroendocrine tumor (NET). PLoS One. 2016; 11: e0158140.

16. Yao JC, Hassan M, Phan A, Dagohoy C, Leary C, Mares JE, Abdalla EK, Fleming JB, Vauthey JN, Rashid A, Evans DB. One hundred years after carcinoid: epidemiology of and prognostic factors for neuroendocrine tumors in 35,825 cases in the United States. J Clin Oncol. 2008; 26: 3063-3072.

17. Yao JC, Shah MH, Ito T, Bohas CL, Wolin EM, Van Cutsem E, Hobday TJ, Okusaka T, Capdevila J, de Vries EG, Tomassetti P, Pavel ME, Hoosen S, et al. Everolimus for advanced pancreatic neuroendocrine tumors. N Engl J Med. 2011; 364: 514-523.

18. Yao JC, Fazio N, Singh S, Buzzoni R, Carnaghi C, Wolin E, Tomasek J, Raderer M, Lahner H, Voi M, Pacaud LB, Rouyrre, Sachs C, et al. Everolimus for the treatment of advanced, non-functional neuroendocrine tumours of the lung or gastrointestinal tract (RADIANT-4): a randomised, placebo-controlled, phase 3 study. Lancet. 2016; 387: 968-977.

19. Gross S, Rahal R, Stransky N, Lengauer C, Hoeflich KP. Targeting cancer with kinase inhibitors. J Clin Invest. 2015; 125: $1780-1789$. 